\title{
Equipos de alto desempeño para la competitividad de empresas de servicios industriales
}

\author{
High-performance teams to ensure the competitiveness of \\ industrial service companies \\ María-Elena Díaz-Calzada \\ Universidad Autónoma de Querétaro, Santiago de Querétaro, México. \\ Josefina Morgan-Beltrán (iD \\ Universidad Autónoma de Querétaro, Santiago de Querétaro, México. \\ Adriana-Quizuani Arredondo-Morales \\ Universidad Autónoma de Querétaro, Santiago de Querétaro, México.
}

\begin{abstract}
Resumen
Objetivo: este estudio busca proponer elementos base para el desarrollo de equipos de alto desempeño, presentando un diagrama como propuesta para el desarrollo de un equipo dentro de la industria. Método: se realizó bajo una metodología cuantitativa utilizando la técnica de encuestas y el análisis de documentos. Resultados: se encontraron tres principales elementos: capacitación, comunicación y organización, en el cual se evidenció que es importante desarrollar el talento humano quien forma parte de la maduración del equipo de trabajo para lograr su eficacia. Conclusiones: Asimismo, es esencial gestionar el conocimiento que se crea al interior del equipo para difundirlo a través de la capacitación y retroalimentación. Uno de los elementos para el éxito de una empresa es su capacidad para crear y utilizar el talento de sus integrantes.
\end{abstract}

Palabras clave: Capital Humano, gestión del conocimiento, desempeño organizacional.

Clasificación JEL: M, M1, M12.

\section{Abstract}

Autor de

Correspondencia

jmorganbeltran@yahoo.com.mx

Recibido: $21-01-2020$

Aceptado: 07-04-2020

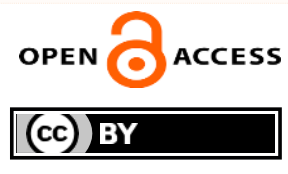

Copyright (C) 2020

Desarrollo Gerencial
Purpose: To propose basic elements for the organization of high-performance teams, presenting a diagram for the development of teams within the industry sector. Method: A quantitative research was conducted, which involved performing document analysis and employing instruments such as the administration of surveys. Results: Three main elements were identified: training, communication, and organization. It was found that it is fundamental to harness human talent as it is essential to the maturity of the engagement team and contributes to the achievement of efficiency. Conclusions: It is important to manage the knowledge created within the team to transfer it through training and feedback. A key element in a company's success is its capacity to create and put its members' talent to use.

Keywords: Human capital, knowledge management, organizational performance.

JEL Classification: M, M1, M12.

Como citar este artículo (APA):

Díaz-Calzada, M., Morgan-Beltrán, J. y Arredondo-Morales, A. (2020). Equipos de alto desempeño para la competitividad de empresas de servicios industriales. Desarrollo Gerencial, 12(1), 1-19. https://doi.org/10.17081/dege...3685 


\section{Introducción}

A partir de los años 90, con el surgimiento del concepto de la Gestión del Conocimiento, el capital humano retomó mayor importancia en las organizaciones y se convirtió en activo intangible medido por el conocimiento, la creatividad y la innovación de sus empleados. En este sentido, se considera que el éxito de una empresa depende de su capacidad para crear y utilizar el talento de sus integrantes, ya que el desempeño de cualquier tipo de organización no solo debe ser medido en su parte financiera, sino también en la gestión de su capital humano.

Por ello, partiendo de la problemática que genera la inquietud de analizar de qué forma pueden formarse equipos de alto desempeño que sean auto-dirigidos y aporten a la organización sus conocimientos y experiencias, se formuló la pregunta que guió la presente investigación: ¿De qué forma se pueden desarrollar equipos de trabajo de alto desempeño que permitan incrementar la competitividad en las organizaciones?

El objetivo del presente estudio se basó en proponer elementos claves para el desarrollo de equipos de trabajo de alto desempeño, presentando un diagrama como propuesta para el trabajo en equipo dentro de las empresas de servicios industriales. Para el desarrollo de la investigación, se realizó una metodología cuantitativa en la cual se utilizó el análisis de documentos y la aplicación de encuestas al personal de nómina directa de dos plazas, equipo de trabajo de Apodaca Nuevo León y de Santiago de Querétaro, México.

Para lo anterior, se consultó la forma en que las organizaciones desarrollan sus equipos de trabajo de alto desempeño; para esto, teniendo en cuenta los facilitadores de la gestión del talento de Carrión (2007) se evaluó la manera en el que el capital humano puede desarrollarse, además, considerando los elementos requeridos para la formación de equipos de alto desempeño de Robbins y Judge (2013) se analizó las tres áreas de eficacia de un equipo. Así como también el contexto, composición y proceso que llevan al avance de los equipos y las estrategias que utilizan las organizaciones para gestionar el conocimiento, según lo indicado por Valhondo (2003).

Por otra parte, se identificaron cuáles fueron los equipos de alto desempeño que permiten la gestión del conocimiento dentro del mismo, por medio de las etapas: evaluación, infraestructura, análisis, diseño y desarrollo, sistema de gestión de conocimiento, despliegue y evaluación. Asimismo, se categorizó la respuesta de los integrantes de cada equipo en cada reactivo, atendiendo los indicadores empíricos establecidos, separando los resultados de los dos equipos, los cuales se presentaron en valores porcentuales y en opiniones cualitativas. 
Los tres principales elementos encontrados en las respuestas de los dos equipos fueron: capacitación, comunicación y organización. Se encontró que es importante desarrollar el talento humano el cual forma parte de la maduración del equipo de trabajo para lograr su eficacia, que a su vez es esencial para gestionar el conocimiento que se crea y se difunde al interior de estos logrando así la capacitación y la retroalimentación del mismo.

\section{Fundamentación teórica}

\section{Capital Humano}

La manera en que se les ha ido dado importancia a las personas dentro de las organizaciones ha cambiado a través del tiempo, autores como Suárez (1992), señalan que el capital humano es el valor del conocimiento que poseen los individuos tanto de un país como de una empresa. Esta es una capacidad creativa que se refleja en hechos que dan resultados, como por ejemplo la calidad en los servicios.

La gestión del capital humano es contemplar la administración de todos los elementos que contribuyen al desarrollo y manejo aplicado de las competencias humanas de una manera productiva, considerando tanto la cultura, misión, visión como la estrategia de la organización, sin perder de vista un ambiente laboral digno en el cual se pueda dar el desarrollo humano básico (Martínez y Corrales, 2011). El éxito de toda organización radica en la habilidad que demuestra al trabajar bien con y a través de otros (García, 2012).

Por otro lado, para Carrión (2007), el capital humano es uno de los tres capitales que forma parte del capital intelectual y consiste en todo el conocimiento explícito o tácito de las personas en conjunto con su capacidad para crearlo por lo cual involucra su capacidad de aprendizaje. Asimismo, para este autor es la base de los otros dos capitales; el capital estructural y el capital relacional. El capital estructural es el conocimiento que está en las personas, pero la empresa logra exponerlo, sistematizarlo y agregarlo volviéndose propiedad de la empresa. El capital relacional son todas las relaciones que tiene la empresa con su medio. Stewart (1998) mencionó que el capital intelectual es donde se integra conocimiento, experiencia, material y propiedad intelectual, la cual da un valor agregado a futuro y es parte de un activo intangible de una empresa.

Bungay (2012), por su parte, señala que, en el desarrollo de las personas, cuando se manifiesta que un individuo está preparado es cuando se ha entendido qué hacer, sin embargo, para actuar se debe tener disposición y capacidad para ejecutarlo. Es por ello que enuncia que las empresas deben proporcionar los medios necesarios. Existe una diferencia entre estar preparado, estar dispuesto y ser capaz. Con base en la importancia que tienen los equipos en el éxito de una empresa, Guízar (2013) 
María-Elena Díaz-Calzada, Josefina Morgan-Beltrán, Adriana-Quizuani Arredondo-Morales

afirma que existen herramientas que permiten detectar el grado de preparación de la empresa para formar equipos de trabajo.

Además, Carrión (2007), señala que el talento es dado por el alto rendimiento que se obtiene de los resultados de las actividades de una persona o un grupo de ellas comprometidas a alcanzar las metas. En este aspecto, definió dos tipos de talentos: el talento individual, que es aquel que posee una persona y el organizativo que se da en un equipo de trabajo. El talento siempre va a depender de la organización y su entorno y consta de tres factores: capacidad, compromiso y acción.

Para definir que el talento no es innato y que este se puede desarrollar, Carrión (2007) basado en Cubeiro contempló los siguientes requisitos:

- Motivación, considerando que se debe disfrutar lo que se hace

- Herramientas de conocimiento que permitan desarrollar las competencias con las que se cuentan

- Generación de nuevos hábitos a traves de nuevas acciones de beneficio

- Disponibilidad de recursos

De esta forma, se puede afirmar que el talento de la organización proviene de la selección y capacitación de las personas que la integran y de la conformación de un entorno organizativo y motivante que favorezca el compromiso con la organización, para ello, existen facilitadores de la gestión del talento, sin embargo, resulta complicado delimitar el comportamiento de las personas en las organizaciones ya que las empresas están relacionadas con las personas y para operar dependen de ellas para alcanzar los objetivos. Las organizaciones nacen con la intención de aprovechar la unión de los esfuerzos de las personas al trabajar en conjunto. Es importante la capacidad de las empresas para desarrollar y utilizar las habilidades intelectuales y las competencias individuales de sus miembros (Chiavenato, 2009).

Actualmente, las organizaciones orientan sus esfuerzos primeramente en sus procesos y luego a las tareas, es decir se está más atento en los fines y resultados que en los medios. Por tanto, se está enfocando de forma holística, en el todo y no en sus partes, ya no en puestos individualizados, separados y confinados, sino en el trabajo en conjunto que se realiza en equipos autónomos y multidisciplinarios (Chiavenato, 2009).

Autores como Suñe, Gil, y Arcusa (2013), exponen que aprender es un proceso dinámico que encaja con el dinamismo de las organizaciones; este concepto se adapta en diferentes niveles individual, grupal y organizacional por lo que gestionar el talento humano se convierte en un concepto clave para el éxito de las organizaciones.

Por otra parte, existen ciertos tipos de personas que tienen algún tipo de diferencial competitivo que hacen ser valoradas y esto hace que se les denomine talento. Según Chiavenato (2009), dentro del 
María-Elena Díaz-Calzada, Josefina Morgan-Beltrán, Adriana-Quizuani Arredondo-Morales

talento se contemplan cuatro aspectos importantes; conocimiento que es el saber y constante aprendizaje; habilidad, en el cual se encuentra el saber hacer, es decir, donde se aplica el conocimiento; juicio, la capacidad de análisis para poder priorizar y actitud donde el hacer que ocurra algo lleva a la persona a su máximo potencial.

En cuanto al desempeño organizacional, Steiner (2012) menciona que el desempeño de una empresa no solo se debe medir en la parte financiera, sino que también debe considerarse aspectos como el conocimiento, la creatividad e innovación los cuales dan un efecto positivo en los resultados económicos.

\section{Gestión del conocimiento}

Molina y Marsal (2001) señalan que cuando se habla de gestión del conocimiento se incluye el Knowhow de la empresa, es decir el capital intelectual, el interés de compartir el conocimiento (cultura organizativa) y los elementos necesarios para lograr el acceso al conocimiento como la tecnología. Asimismo, Canals (2003) menciona que la gestión del conocimiento es la función de optimizar el elemento conocimiento, el cual lo define como la cualidad de una persona para interpretar su entorno y la posibilidad de actuar. Para este autor, la acción de gestionar el conocimiento implica las condiciones que facilita el intercambio de conocimiento, ya que le permite a una organización actuar con base en su entorno lo que implica un agregado a la suma de lo que logra cada persona, además señala que la gestión del conocimiento se divide en dos procesos básicos; la creación del conocimiento y la transmisión del mismo y que los grupos son parte fundamental de este.

La teoría de la creación del conocimiento organizacional de Nonaka y Takeuchi (1999), expone un proceso en espiral de la conversión del conocimiento, el cual está integrado por cuatro etapas:

- Socialización: es el medio por el cual se da el intercambio del conocimiento tácito, implícito sin comunicación verbal

- Exteriorización: es la codificación del conocimiento tácito a un lenguaje de manera que se pueda entender por otro

- Combinación: genera conocimiento codificado (explicito) por conocimientos exteriorizados por socialización

- Interiorización: es el medio donde el conocimiento explicito se transforma en tácito por práctica.

Asimismo, Schamer (2001) citado en Oltra (2002) determina tres fases de la gestión del conocimiento: las TIC y el conocimiento explicito, la creación del conocimiento-que es la interacción entre conocimiento tácito y explícito- y la evolución del conocimiento tácito. También cita a Sveiby quien afirma que la gestión del conocimiento es la mezcla de tres orígenes; el americano, en el ámbito de la información y la inteligencia artificial: el japonés, en la creación y la innovación; y el sueco en la medición y la estrategia, quien a su vez también plantea tres fases por las cuales pasó la evolución de gestión del conocimiento, 
María-Elena Díaz-Calzada, Josefina Morgan-Beltrán, Adriana-Quizuani Arredondo-Morales

como primera fase delimitó entre los años 1985 y 1990, argumentando que solo se tenía interés en las capacidades y habilidades de las personas para generar conocimiento. La segunda fase la marcó entre 1991 y 1997, en ella manifestó que con la aparición de las tecnologías de la información la internet causó revuelo en los procesos de gestión de la industria, además aparecieron los términos gestión del conocimiento y capital intelectual. La tercera fase, que es la actual, comienza a considerar al ser humano como factor principal en la creación e innovación del conocimiento. Altvater y Mahnkopf (2002) comentan que con las tecnologías de la comunicación resulta más fácil codificar el conocimiento, y de esta forma el conocimiento se transforma en un valor económico agregado para la empresa.

Huysman (2010) presenta la comparativa de dos términos, en el caso de la organización que aprende, para que se pueda dar el aprendizaje, la ejecución de tareas podría considerarse como las pruebas que se están realizando, y en el de caso de aprendizaje organizacional, se considera a la formalización o maduración del proceso que se llevó a cabo y el estudio de lo emergente, así como también la evolución de la organización, por lo tanto, se puede afirmar que ambos términos están ligados.

Por otra parte, para Oltra (2002), la gestión del conocimiento se integra en conjunto con Tecnologías de la Información y Comunicación (TIC), enfoque humano y capital intelectual. Afirma que existen dos elementos comunes entre las definiciones que se han presentado para definir gestión del conocimiento; primero, señala los procesos organizativos, donde el elemento principal es el conocimiento y la gestión del conocimiento como un grupo de políticas claramente analizadas. Dentro de la gestión del conocimiento se contempla la integración de políticas como una herramienta para perfeccionar el conocimiento dentro de la organización como un recurso agregado. El área de implementación puede ser muy diversa como por ejemplo: procedimientos de medición de activos intangibles, tecnología de la información y comunicación con sistemas de gestión de la información, sistemas de gestión de personas, así como la mezcla de diferentes áreas.

Asimismo, de acuerdo con López, Montes y Vázquez (2003), la gestión del conocimiento también promueve la innovación ya que se incluyen una gran cantidad de actividades en las empresas que de alguna manera contribuyen en generar nuevos conocimientos tecnológicos o mejorar los existentes y todo esto se aplica a la obtención de nuevos bienes, servicios, procesos y demás.

Oltra (2002), nuevamente define a la gestión del conocimiento como "el conjunto de políticas deliberadas que plantea la dirección de la organización con el objeto de optimizar la utilidad del conocimiento como recurso estratégico de la organización" (p. 63). Donde considera que el objetivo de la gestión del conocimiento de la dirección es lograr el aprendizaje organizativo, manipulándolo hacia la dirección correcta. Chris (1999) menciona que las empresas son capaces de mejorar habilidades para un aprendizaje productivo, sin embargo, no considera investigar y/o mejorar la implantación de las mismas para que sean efectivas. 
Cohen y Levinthal (1990) exponen que la capacidad de absorción es reconocer información externa que sea de valor y procesarla para poder aplicarla con fines productivos, lo cual puede considerarse como parte de la innovación. Conforme los conocimientos se incrementan, mejora la capacidad de adquirir conocimiento nuevo, así como la capacidad de tenerlo presente y aplicarlo. Oltra (2002), fusionó la diversidad de campos que influyeron y están directamente relacionados con la gestión del conocimiento para dar una definición más completa.

Finalmente, la gestión del conocimiento:

Aglutina el conjunto de políticas deliberadas que plantea la dirección con el objeto de optimizar la utilidad del conocimiento como recursos estratégicos de la organización, facilitando la consecución de los objetivos de la misma y el sostenimiento de sus ventajas competitivas. En este sentido, cobran especial relevancia las intervenciones dirigidas a aumentar la eficacia del proceso general de aprendizaje organizativo en todos sus niveles: grupal y de la organización en su conjunto (Oltra, 2002, p. 225)

Además, aseguró que la gestión del conocimiento abarca la mayor parte de las actividades de la empresa, su plan de acción abarca un todo enfocado en un objetivo, que el conocimiento sea una utilidad estratégica en la organización. Propone que la gestión del conocimiento como una referencia de la dirección de empresas dentro de la nueva economía y el nuevo estilo de las organizaciones, es decir, las organizaciones del conocimiento. (Oltra, 2002)

Asimismo, Tovar y Morgan (2017), señalan que el conocimiento comienza en las personas y se transmite a los demás al interior de la organización para después enviarse al exterior de la organización cuando aplica. El conocimiento tácito que tienen en las empresas es necesario hacerlo explicito hacia los demás miembros de la organización, las personas tienen conocimientos por todas sus experiencias obtenidas durante su vida laboral y con el apoyo Institucional se hace más fuerte y sólida está transferencia del conocimiento que se genera en las organizaciones

Valhondo (2003) comenta que, en el alineamiento de la gestión del conocimiento con la estrategia del negocio, la base es el conocimiento, a partir del cual se crea la estrategia y esta a su vez orienta a la gestión del conocimiento, para ello, es importante la conexión entre los altos niveles donde se desarrollan las estrategias y quienes tienen que ser muy específicos para permearla hacia los niveles de abajo donde se realiza el desarrollo del sistema. Para poder sincronizar la estrategia y el conocimiento es necesario conocer el déficit que se tiene de ambos. 


\section{Equipos de Alto desempeño}

Alcover y Gil (2002) mencionan que ante el contexto dinámico de las organizaciones la ganancia, creación y actualización del conocimiento son elementos básicos de las estrategias necesarias para construir equipos de alto desempeño, sin embargo, Choque y Pérez (2013) afirman que la problemática en las empresas comerciales es la falta de herramientas, procesos y mecanismos de control por lo que no se cuenta con información adecuada para la toma de decisiones, así como cumplimiento de procedimientos, normas y políticas, y esto es importante considerarlo para la elaboración de la estrategia.

Asimismo, Thompson, Peteraf, Gamble, y Strickland (2012) señalan que llevar a cabo una estrategia se requiere de trabajo en equipo y no se debe considerar que sea sólo de altos administrativos; manifiestan además que un grupo de recursos (recurso humano, recurso intangible) es una integración de activos enfocados a una o varias actividades multifuncionales. Los equipos de trabajo son un conjunto de dos o más individuos, entre ellos son interdependientes en sus tareas, interactúan, se adaptan y comparten la responsabilidad de los resultados al igual que la meta en común. Los grupos de trabajo han sido considerados como un instrumento administrativo (Mendoza y Zambada, 2006).

Topchik (2008) define que un equipo de trabajo es un grupo de personas que con su trabajo interdependiente alcanzan los objetivos, esto teniendo una buena comunicación y tomando decisiones. Plantea que los equipos de trabajo cuentan con un grado de autonomía a parte de lograr plantear un propio proceso que les ayuda a lograr sus metas.

De esta manera, se puede definir a un grupo como "dos o más individuos que interactúan, que son interdependientes y que se reúnen para lograr objetivos específicos" (Robbins y Judge, 2013, p. 273). En este sentido, Robbins y Jugde (2013) propusieron un modelo de cinco etapas de desarrollo de un grupo, las cuales clasifico en: formación, tormenta, normatividad, desempeño y suspensión. Doz y Kosonen (2010) destacan que desarrollar talentos clave apoya el desarrollo de un grupo.

Nelson (2001), asegura que, como miembro de un equipo, es importante que el integrante tenga iniciativa, se involucre en las actividades, se integre, se comprometa, participe en las metas del equipo, agregar valor con las habilidades propias y responsabilidad en el crecimiento del equipo, ya que según Daft y Marcic (2010) los equipos son auto-dirigidos y provienen de equipos de solución de problemasestos son equipos que se encargan de revisar la calidad, la eficiencia y el ambiente de trabajo de una misma área, y por lo general están formados de cinco a 12 personas-. Cuando estos grupos progresan llegan a tener una gran cantidad de habilidades, pueden llegar a ser equipos más grandes de cinco hasta 20 personas, en este caso los empleados se rotan de puesto para cumplir una tarea, siendo supervisados por una persona electa y convirtiéndose en un equipo auto-dirigido. 
María-Elena Díaz-Calzada, Josefina Morgan-Beltrán, Adriana-Quizuani Arredondo-Morales

Goleman (2014), denominó flujo grupal, a aquel equipo que logra con éxito el trabajo en conjunto y se supera a sí mismo. Este autor analizó a diferentes gerentes exitosos y llegó a las siguientes características:

- Es necesario buscar metas demasiado grandes para dar tiempo a que todo el equipo se pueda alinear a ella, ante un gran desafío surgen los mejores esfuerzos

- La presencia de lealtad en el grupo. Es preciso la formación de vínculos que permitan el propicio de la integración

- Variedad de talentos que propicie una diversidad de habilidades con las cuales se pueda enfrentar fácilmente al entorno cambiante

- Plena colaboración entre el equipo. Existe el soporte entre los integrantes

- Concentración y efusión para lograr los objetivos, enfoque en los resultados

- La consideración de realizar un trabajo que permita una recompensa interior emocional, como un trabajo divertido y fructífero.

Katzenbach y Smith citado en Hawkins (2012) definen equipo como:

Un reducido grupo de personas con destrezas complementarias, comprometidas con un objetivo común, un conjunto de metas de rendimiento y un enfoque compartido por los que se sienten recíprocamente responsables. El enfoque común necesita incluir formas efectivas de reunirse y comunicarse que eleven la moral y el alineamiento, interconectándose eficazmente con todos los grupos de interés clave y de modo tal que los miembros y el equipo aprendan y se desarrollen continuamente (p.52).

Por su parte Palomo (2012) definió equipo de alto rendimiento como:

Conjunto de personas que poseen talento y competencias complementarias y que trabajan para conseguir un objeto común mostrando un alto nivel de compromiso. Interaccionan entre sí aceptando ciertas normas y compartiendo emociones, participando de un sentimiento común llamado espíritu de equipo y alcanzando un alto desempeño y excelentes resultados (p.140).

En este sentido, un equipo de alto rendimiento está comprometido con sus propósitos, objetivos, enfoques y con cada uno de los integrantes. La mejor manera de entender un equipo es observándolo; de allí que Katzenbach y Smith (1996) manifiestan que cuando no se guía disciplinadamente a un equipo, se pierde el potencial de rendimiento.

Un equipo surge a partir de un reto de rendimiento, este es el objetivo primario mientras él sea el medio y no el fin. Existen distintos retos para distintos tipos de equipos, por ejemplo, para los equipos de 
María-Elena Díaz-Calzada, Josefina Morgan-Beltrán, Adriana-Quizuani Arredondo-Morales

producción su reto puede ser desarrollar habilidades, los equipos que asesoran su reto puede ser acordar sus recomendaciones, los equipos que gestionan se pueden enfrentar a problemas jerárquicos.

Singer (2009), subrayó que es importante que la persona que se seleccione para integrar un equipo tenga un talento que genere valor al puesto que desempeñará. Para esto, identificó seis características de un buen integrante tales como: energía, trabajo por ganar, capacidad de aceptación que alguien más gane, asume responsabilidades, disponibilidad y compromiso, y talento o habilidad que agregue valor.

Katzenbach y Smith (1996), proponen que el individualismo no se interponga con el rendimiento del equipo, comenta que la auto preservación y la responsabilidad individual guiadas de manera positiva pueden llegar a ser una fortaleza sumada y que en el momento que cada individuo contribuye este se distingue.

En este mismo orden de ideas, González (2015), sugirió seis etapas para el proceso de un equipo:

- Introducción: en esta etapa los integrantes indagan en que trabajarán, que objetivo persigue el equipo, aquí la información suministrada debe ser clara. Se reconocen las capacidades, valores, intereses, necesidades, actitudes e interrelaciones.

- Creación y dependencia: Se dan las instrucciones para realizar los trabajos de parte de un líder quien debe ser claro y ocuparse de la dinámica del equipo para encaminarlos a su desarrollo y lograr los objetivos.

- Proceso de organización y establecimiento de la estructura: es el seguimiento de los requerimientos necesarios para el logro de los objetivos. Se define la estructura y reglas.

- Proximidad madura y contra dependencia: Los integrantes plantean necesidades e inconformidades, cada miembro tiene definido su papel por lo que solicita los apoyos necesarios y su reconocimiento.

- Independencia y producción: existe la integración del equipo, se tiene claro el objetivo del mismo.

El equipo agrega valor a las normas, los sistemas y los procedimientos.

- Evaluación y mantenimiento: es importante que los integrantes respeten los elementos establecidos en la realización de las tareas para lograr los objetivos, y realizar una evaluación de las actividades. En este punto cada integrante es consciente de su capacidad como integrante y como equipo permitiéndole generar nuevas maneras de eficiente su trabajo.

Finalmente, Robbins y Judge (2013), definieron que la eficacia del equipo hace referencia a su productividad, desempeño asignado por gerentes y la satisfacción de los integrantes. Dentro del contexto se encuentran los recursos adecuados, que son los soportes de la organización hacia el equipo, como la facilidad de información, materiales y herramientas adecuadas, personal correcto, estímulos y administración apropiada. 
En el liderazgo y la estructura se define el funcionamiento del equipo, si la carga de trabajo está dividida entre todos los integrantes, es necesario que el líder y los integrantes estén de acuerdo en las especificaciones del trabajo y la integración de las habilidades individuales para lograr el funcionamiento del equipo. Dentro de los equipos eficaces existe un clima de confianza ya que al confiar entre los mismos miembros y sus líderes facilita el enfoque en el trabajo. En la evaluación del desempeño y sistemas de recompensas, es importante tener en cuenta la evaluación y recompensa de los integrantes considerando la contribución individual y grupal.

\section{Método}

Este estudio se realizó siguiendo una metodología cuantitativa, en la cual se utilizó la técnica de encuestas a 30 empleados incluyendo personal técnico, supervisores y directivos de las 2 sucursales de una compañía del sector industria, con el fin de obtener un acercamiento a la posible respuesta a la pregunta ¿De qué manera se pueden formar equipos de alto desempeño que permitan incrementar la competitividad en las organizaciones?

Para lo anterior, se tomó como muestra a 30 empleados del personal fijo en nómina personal de los niveles: técnicos, supervisión y directivo de ambas sucursales de la compañía, en donde se aplicó una encuesta integrada por 29 reactivos 29 como parte de la investigación para el desarrollo de equipos de alto desempeño a los equipos de trabajo de la compañía HI, empresa que fundada en el 2005 en Hermosillo, Sonora, México y dirigida por HVAC (Heating, Ventilation and Air Conditioning). Las encuestas fueron aplicadas a 28 empleados ya que 2 de ellos se encontraban con incapacidad médica.

Para el trabajo de campo, primeramente se revisó la documentación oficial de la empresa, posterior a ello, se aplicaron las encuestas y finalmente para el análisis de los datos se utilizó el sistema de análisis de encuestas SPSS 2016.

\section{Resultados}

Los resultados que se presentan en esta sección consideran a las 2 sucursales objeto de estudio y se nombran a la primera compañía como empresa $A$ ubicada en el Norte del País y la segunda compañía como empresa $B$ ubicada en el centro del país, esto teniendo en cuenta que existen diferencias culturales a pesar de pertenecer al mismo país.

- $\quad$ El 83\% de los empleados en el equipo de la empresa A (Apodaca Nuevo León), respondió que de alguna forma la empresa los estimulaba a progresar y colaborar, mientras que para el de ellos manifestó que no existía ningún tipo de estímulo. En el equipo de la empresa B (Santiago 
María-Elena Díaz-Calzada, Josefina Morgan-Beltrán, Adriana-Quizuani Arredondo-Morales de Querétaro, Querétaro), el 88\% de sus empleados afirmaron que existe algún elemento en que la empresa estimulaba al equipo a progresar y colaborar, mientras que el $12 \%$ restante no respondió.

- En ambos equipos de trabajo, se detectó que la forma de transmitir los conocimientos, procedimientos y técnicas de trabajo para la compresión de tareas fue principalmente de manera verbal o experiencia de día a día, con un $72 \%$ en la empresa $\mathrm{A}$ y un $58 \%$ en la empresa B respectivamente. La supervisión en la empresa B, señaló la existencia de un manual de inducción, en el cual se hace que el personal nuevo que ingrese lea y firme el manual. Sin embargo, con base a las respuestas obtenidas se detecta una falta de procedimientos, manuales $\mathrm{u}$ otros elementos de información donde se den a conocer los conocimientos hacia los integrantes de los equipos de trabajo.

- En el enfoque de la inversión de recursos para el equipo $A$, el 53\% consideró que si contaba con los elementos necesarios para su trabajo y en el equipo B el $83 \%$ aseguró que si contaba con lo necesario.

- Con respecto al liderazgo, dentro del equipo A un 40\% de los empleados lo consideró bueno y excelente, el $60 \%$ lo consideró con alguna deficiencia donde manifestó la falta de comunicación. Por otro lado, en el equipo B el $84 \%$ lo consideró muy bueno y eficiente y un $16 \%$ mencionó la existencia de ambigüedad por lo que podría existir una falta de decisión, seguridad y firmeza.

- Con relación al reconocimiento, en el equipo A, el 53\% de su personal aseguró no haber obtenido algún reconocimiento por parte de la empresa, y en el equipo B un 40\% comentó no haber tenido.

- Dentro del equipo A, se encontró que el 44\% no conocía algún sistema de control y dirección. En el caso del equipo B el 100\% mencionó comentarios de procedimientos para llevar a cabo las tareas como toma de bitácoras, listados y control.

- En ambas plazas el $80 \%$ aseguró contar con una buena relación con sus jefes y compañeros de trabajo y un $20 \%$ respondió que a veces mantenían buenas relaciones.

- Se observó que el $85 \%$ del equipo A realizó una buena observación del ambiente de trabajo y en el caso B el $100 \%$ comentó positivamente.

- Considerando que el $71 \%$ y el $41 \%$ de los integrantes de los equipos B y A respectivamente mencionaron que los programas de mantenimiento y actividades del mismo son el principal elemento para el logro de los objetivos, se encuentra la razón de la demanda de capacitación.

- En los sistemas de evaluación se detectó que el 73\% de los integrantes del equipo A indicó desconocerlo, que no existían o no contestó a la pregunta y un $27 \%$ argumentó que era por supervisión directa. En el caso del equipo B, el 57\% aseguró que por medio de exámenes y habilidades desarrolladas y un $43 \%$ comentó desconocerlo. 
María-Elena Díaz-Calzada, Josefina Morgan-Beltrán, Adriana-Quizuani Arredondo-Morales

- La manera en que se realiza la división de tareas en los equipos, para el caso de $A$, el $40 \%$ indicó que el supervisor era quien asignaba las tareas a realizar y $13 \%$ que estas se dividían cooperativamente entre el equipo, para el caso B, el 50\% mencionó que estas se dividen en el mismo equipo y el $33 \%$ comentó que estas son programadas y asignadas.

- Se consultó si se reconocían los números de integrantes que participaban en cada uno de los equipos. En el caso B, se mencionaron dos cifras distintas. En el equipo A se detectó una diferencia en las respuestas de los equipos de cada área. En la clasificación por área, en el área de administración y soporte a la operación se detectaron dos cifras, en el área de HVAC se detectaron tres cifras distintas, en infraestructura cuatro cifras y en proyectos el resultado se dividió en tres respuestas diferentes.

- Se consultaron a ambos equipos el objetivo de HI, la visión y misión donde se detectaron distintas ideas. Para el caso A, el 47\% de los integrantes desconocía el objetivo de la empresa y $46 \%$ mencionaron objetivos distintos a los de la empresa. Para el caso B, el $83 \%$ de los empleados comentaron desconocer o no recordar los objetivos, misión y visón de la empresa, sólo el $17 \%$ mencionó un objetivo.

- En el equipo, con respecto a las metas del equipo, el 50\% aseguró dar un trabajo de calidad, $25 \%$ no contestó y el $25 \%$ restante se dividió en distintas respuestas. Para el caso B, el $33 \%$ consideró cumplimiento con las tareas diarias y la realización de trabajos de calidad, el 17\% mencionó mejorar tiempos y desempeño y otro, el $17 \%$ no contestó.

\section{Discusiones}

De los resultados anteriormente planteados, se evidencia que tanto las empresas como los equipos son dinámicos, (Alcover y Gil, 2002). Ante el contexto activo de las organizaciones, la ganancia, creación y actualización del conocimiento son elementos básicos para definir las estrategias, por lo que se planteó crear un ciclo de retroalimentación del conocimiento establecido al interior de los equipos para capacitar y madurar al mismo con diversos elementos.

En la Figura 1, se muestra como primer bloque el desarrollo del talento de los integrantes que forman o formarán parte del equipo, Hawkins (2012) mencionó que es necesario que el equipo este integrado con diferentes destrezas complementarias, variedad de conocimientos técnicos necesarios, capacidades y habilidades; considerando lo indicado por Carrión (2007) donde enuncia que el talento no es innato sino desarrollable se toman en cuenta los elementos para el desarrollo del talento, agregando la inversión de recursos y facilidad de herramientas de conocimiento, los cuales son necesarios para facilitar la capacitación del personal.

En el segundo bloque, se considera el modelo de eficacia del equipo de Robbins y Judge, (2013), donde se contempla la orientación del equipo para lograr su potencial como grupo, generando un 
María-Elena Díaz-Calzada, Josefina Morgan-Beltrán, Adriana-Quizuani Arredondo-Morales

ambiente donde se pueda desenvolver, asegurar que el mismo este integrado por los miembros indicados y que estos puedan desarrollarse tanto grupal como individualmente ya que se convierte en un valor agregado al equipo. Por último, el proceso y proyección que se da al conjunto al interior de la empresa, especificando propósitos y metas específicas.

En el tercer bloque se considera lo mencionado por Stewart (1998), quienes consideran el conocimiento, experiencia, material y propiedad intelectual, como valor agregado, estos son un activo intangible de una empresa, los cuales no están reflejados en los estados financieros, por lo que se integra la gestión del conocimiento considerando las etapas de Valhondo (2003, p. 154), donde la intención es alinear los conocimientos creados, documentarlos y difundirlos de manera que sean efectivos en el momento que se inyectan en el equipo de trabajo, convirtiéndose en necesario; evaluar los conocimientos desarrollados, realizar un diseño (metodología y estandarización) y en caso de ser necesario un pulimiento de lo creado, comunicarlo y difundirlo entre los integrantes del equipo y evaluar los efectos en el mismo.

Figura 1. Elementos propuestos para el desarrollo de equipos de alto desempeño

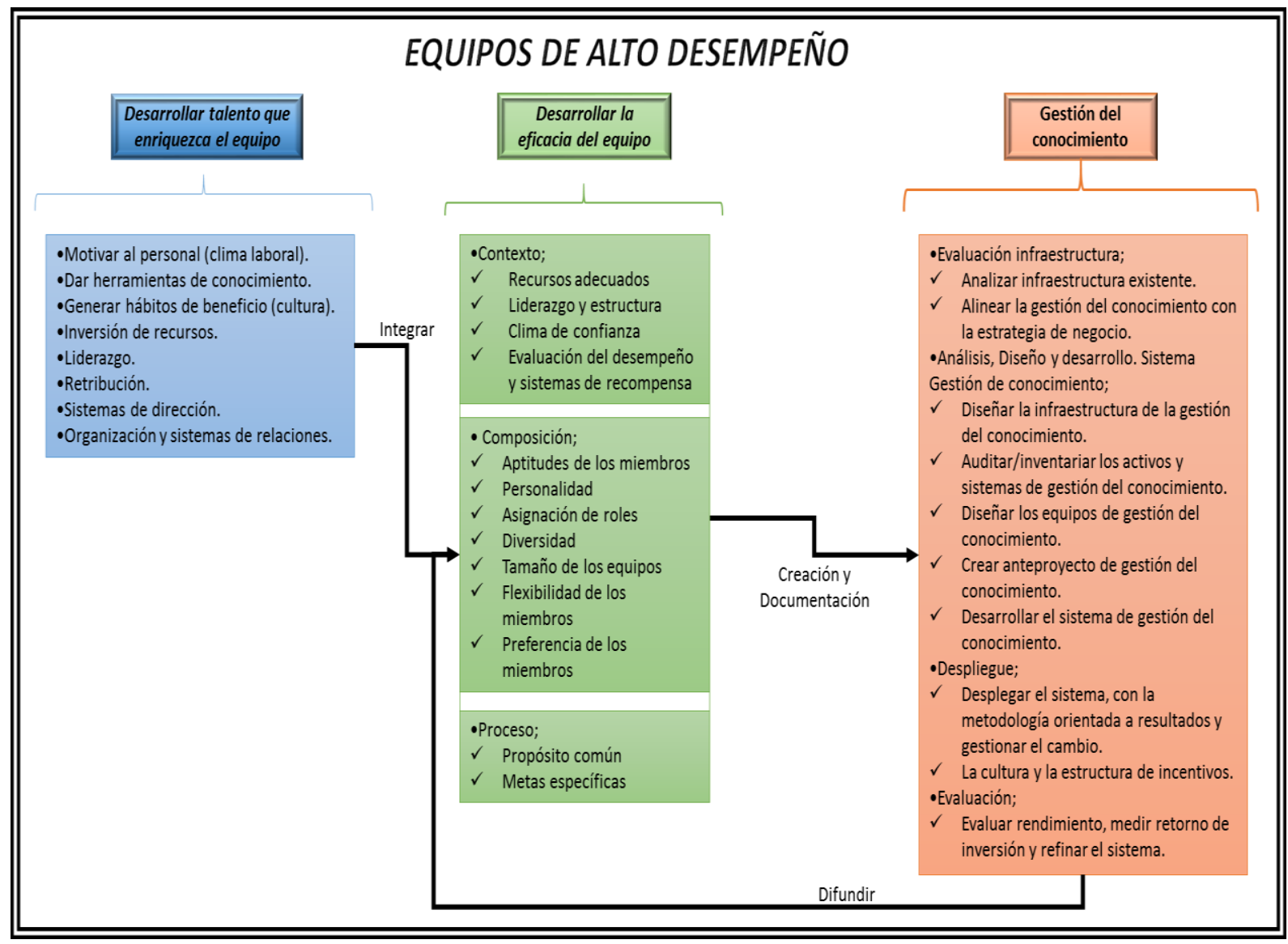

Fuente: Elaboración propia (2019). 
De esta manera y con base en los resultados obtenidos en los equipos de la compañía HI, en la Figura 2 , se proponen los elementos detectados para el desarrollo de sus equipos. Las tres principales áreas de oportunidad encontradas fueron: capacitación, comunicación y organización.

Figura 2. Elementos detectados para el desarrollo de sus equipos

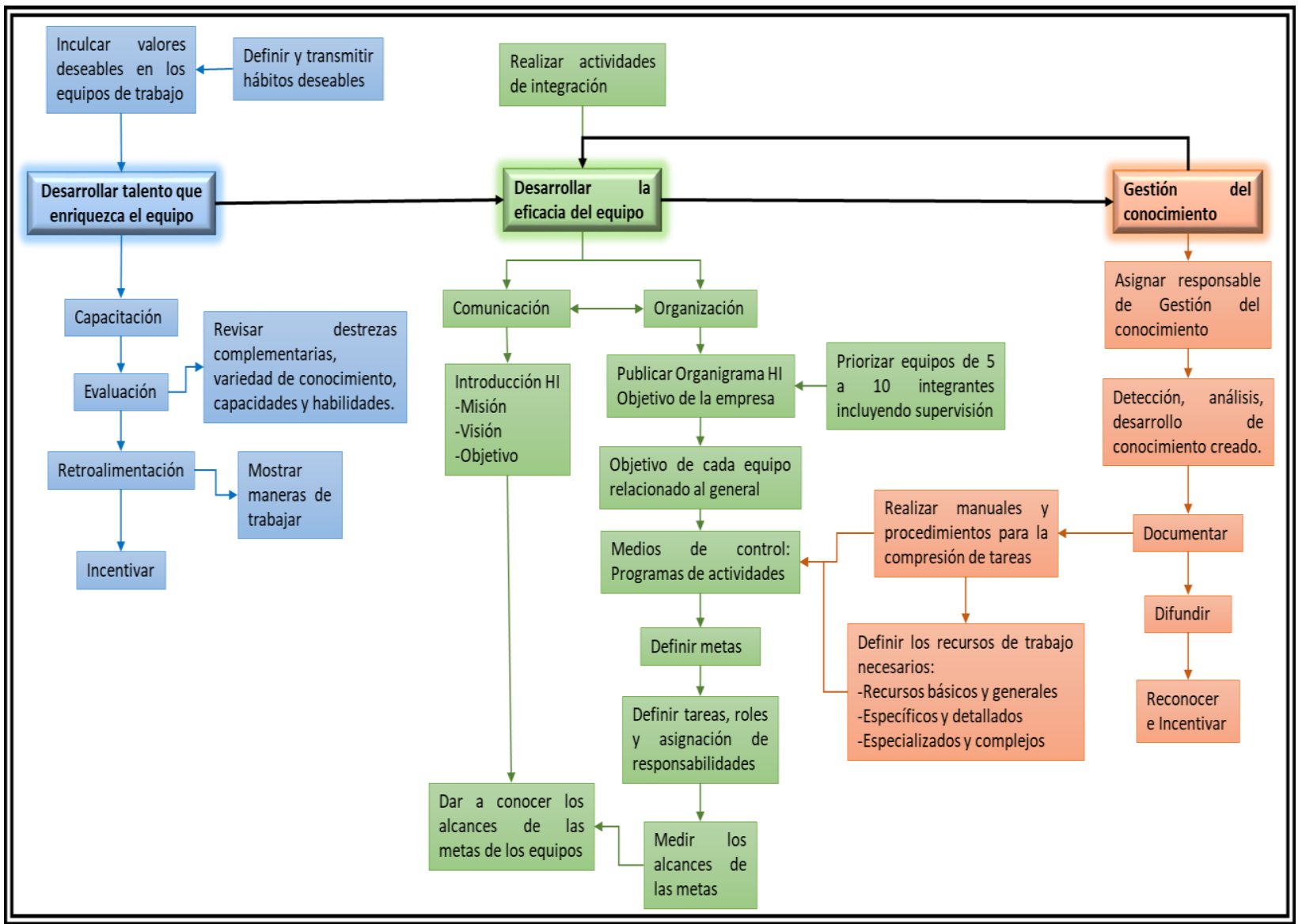

Fuente: Elaboración propia (2019).

Considerando el diagrama de elementos propuestos anteriormente, para el trabajo de equipos de alto desempeño, en la etapa de desarrollo de talento se contempla lo siguiente:

- Capacitación: deberá ser evaluada y complementada con la revisión de la composición de los equipos, analizando las destrezas, conocimientos, capacidades y habilidades de cada uno de los integrantes. Los equipos deben ser retroalimentados con los resultados obtenidos y complementados acciones de mejora, agregando incentivos (económicos, diplomas, felicitaciones, dinámicas, retos, etc.) para propiciar el esfuerzo. Es importante que dentro del desarrollo del equipo de la compañía HI, se agreguen dentro valores y hábitos deseables. 
- Para el desarrollo de la eficacia en el equipo es necesario la comunicación y organización. Se recomienda difundir la misión, visión y objetivos de la empresa, partiendo de lo general para llegar a lo específico con la definición de los objetivos, metas y alcances de cada equipo. Es necesario que la organización de los equipos sean entre cinco a 10 integrantes considerando lo expuesto por (Robbins y Judge, 2013) y (Daft y Marcic, 2010). Además, se debe realizar la publicación del organigrama de la empresa donde se expongan claramente los departamentos, equipos e integrantes de cada área y cada grupo, así como el objetivo general de la compañía y los objetivos de cada equipo relacionados con el general.

- Se expone la necesidad de desarrollo de medios de control donde se integren los programas de las actividades, las metas a alcanzar, definir y asignar; roles, tareas y responsabilidades, medir los alcances obtenidos y comunicarlos tomando como referencia de comparación la meta planteada. Dentro del desarrollo de un equipo es fundamental el trabajo en conjunto, por lo que se propone la utilización de actividades de integración.

- Con relación a la gestión del conocimiento, se propone la asignación de un responsable para gestionar el conocimiento, detectar, analizar y desarrollar el mismo al interior de la empresa. Así como también documentar el conocimiento por medio de manuales y procedimientos, definir los recursos necesarios, socializar con los equipos y reconocer e incentivar a los creadores del nuevo recurso intelectual.

- Por último, desarrollar un equipo eficaz con los integrantes adecuados, documentando los conocimientos creados y aprendidos e inyectándolos de capacitación y entrenamiento va permitir que estos sean eficientes y maduros al momento de tomar sus propias decisiones.

\section{Conclusiones}

Finalmente, en este estudio se concluyó que las principales áreas de oportunidad encontradas en los equipos de la compañía HI, fueron: capacitación, comunicación; definición de canales y formas de difusión, sistemas de establecimiento y clarificación de las metas y objetivos ya que se detectó una gran diversidad de ideas sin tener alguna en común, falta de incentivos, para los cuales se recomienda establecer los elementos que se desean evaluar, desarrollo de evaluaciones y diseño de los parámetros de evaluación, necesidad de gestión del conocimiento, el cual da un valor agregado a la empresa, por lo que el comienzo de la documentación de sus conocimientos puede ser un gran sustento para la capacitación de sus mismos empleados, elemento con mayor demanda según los resultados obtenidos. 
Todo lo anterior apunta a que el trabajo en equipo debe ser una parte fundamental para esta compañía ya que un equipo no puede trabajar solo sino que debe hacerlo en conjunto con los demás para el logro de los objetivos organizacionales. De esta manera, cuando se consigue que un equipo de trabajo es de alto desempeño, es decir alto rendimiento, el compromiso que se obtiene de cada uno de los integrantes es mayor porque no solo se comparte la responsabilidad sino que también se logra el individual y grupal.

\section{Referencias}

Alcover, C. y Gil, F. (2002). Crear conocimiento colectivamente: aprendizaje organizacional y grupal. Revista de Psicología del Trabajo y de las Organizaciones, 18(2-3), 259-301. https://journals.copmadrid.org/jwop/art/4d5b995358e7798bc7e9d9db83c612a5

Altvater, E., \& Mahnkopf, B. (2002). Las limitaciones de la globalización; economía, ecología y política de la globalización. México: Mundo del siglo XXI.

Bungay, S. (2012). Estrategia en acción: Cómo cerrar la brecha entre planes, operaciones y resultados. México: Grupo Editorial Patria S.A.

Canals, A. (2003). Gestión del conocimiento. Barcelona: Universidad Oberta de Catalunya. http://www.uoc.edu/dt/20251/index.html

Carrión, J. (2007). Estrategia de la visión a la acción. (2 .ed.). Madrid: Alfaomega ESIC.

Chiavenato, I. (2009). Gestión del Talento Humano. (3 ${ }^{\text {a }}$.ed.). México: McGrawHill.

Choque-Silva, A., y Perez-Vargas, L. (2013). La auditoria al desempeño en una empresa comercial. [Proyecto de grado Pregrado, Universidad Mayor de San Andrés, Bolivia] Repositorio Institucional RIUMSA. http://hdl.handle.net/123456789/3139

Chris, A. (1999). Sobre el aprendizaje organizacional. México: OXFORD University Press.

Cohen, W., \& Levinthal, D. (1990). Absorptive Capacity: A new perspective on learning and innovation. Administrative Science Quarterly, 35(1), 128-152. https://www.jstor.org/stable/2393553

Daft, R., y Marcic, D. (2010). Introducción a la administración. (6 .ed.).México: Cengage Learning.

Doz, Y., y Kosonen, M. (2010). Agilidad Estrategica. México: Grupo Editorial Patria. 
García, S. (2012, Diciembre). Detección de Competencias en el Personal de Alto Desempeño como Herramienta Estratégica de Selección, Retención, Evaluación en la Organización. Estudio De Caso: Área de Ventas en una empresa comerzializadora de Sistemas de aire comprimido. Querétaro: México. http://ri.uaq.mx/xmlui/handle/123456789/921

Goleman, D. (2014). La inteligencia emocional en la empresa. Buenos Aires: Ediciones Barataria.

González, A. (2015). Desarrollo Organizacional. México: Publicaciones Administrativas Contables Jurídicas S.A.

Guízar, R. (2013). Desarrollo Organizaciónal Principios y Aplicaciones.(4. a ed.). México: McGraw Hill.

Hawkins, P. (2012). Coaching y Liderazgo De Equipos. Buenos Aires: Ediciones Garnica S.A.

Huysman, M. (2010). An organizational learning approach to the learning organization. Europan Journal of Work and Organizational Psychology, 133-145. https://doi.org/10.1080/135943200397905

Katzenbach, J. R., y Smith, D. K. (1996). Sabiduría de los equipos: el desarrollo de la organización de alto rendimiento. Madrid: Editores Díaz de Santos.

López, N., Montes, J., y Vázquez, C. (2003). Fuentes Tecnológicas para la Innovación. Algunos Datos para la Industria Española. Revista Madrid, 6-20. http://www.madrimasd.org/revista/revista20/tribuna/tribuna1.asp

Martínez, C. y Corrales, M. (Coord.). (2011). Administración de conocimiento y desarrollo basado en conocimiento redes e innovación. México: Cengage Learning Editores.

Mendoza-Gómez, J., y Zambada-Flores, R. (2006). Empowerment en grupos de trabajo en organizaciones mexicanas: motivación, trabajo en equipo y desempeño. CIENCIA UANL, 4(4), 393. https://www.redalyc.org/pdf/402/40290410.pdf

Molina, J., \& Marsal, M. (2001). La gestión del conocimiento en las organizaciones. Buenos aires: Libros en Red.

Nelson, B. (2001). 1001 Formas de tomar la iniciativa en el trabajo. Barcelona: Gestion 2000.

Nonaka, I., \& Takeuchi, H. (1999). La organización creadora de conocimiento. México: OXFORD University Press. 
Oltra, V. (2002). Influencias de las políticas de recursos humanos en los procesos de desarrollo y gestion del conocimiento. [Tesis Doctoral, Universitat de València, España]. Repositorio Institucional RODERIC. http://roderic.uv.es/handle/10550/38472

Palomo, M. (2012). Liderazgo y motivación de equipos de trabajo. México: ESIC Editorial.

Robbins, S., \& Judge, T. (2013). Comportamiento organizacional. (15 ${ }^{\mathrm{a}}$.ed.). México: PEARSON

Singer, B. (2009). El ABC para crear un equipo de negocios exitoso. El código de honor invisible que transforma a grupos de personas en equipos campeones. México: AGUILAR.

Steiner, G. (2012). Planeación Estratégica. México: PATRIA.

Stewart, T. (1998). La nueva riqueza de las organizaciones: el capital intelectual [Traducido al español de Daniel Zadunaisky]. Barcelona: Ediciones Garnica S.A.

Suárez, A. (1992). Diccionario de Economía y Administración. Madrid: Mc-Graw Hill.

Suñe, A., Gil, F., y Arcusa, I. (2013). Manual práctico de diseño de sistemas productivos. Madrid: Ediciones Díaz Santos.

Thompson, A., Peteraf, M., Gamble, J., \& Strickland, A. (2012). Administración Estratégica, teoría y casos. (18 ${ }^{\mathrm{a}}$.ed.). México: McGraw Hill.

Topchik, G. (2008). Gerente por primera vez: Como desarrollar a su equipo. Nashville: Thomas Nelson Inc.

Tovar-Pacheco, F., \& Morgan-Beltrán, J. (2017). Metodología para la gestión del conocimiento en las Mipymes proveedoras de autopartes. Desarrollo Gerencial, 9(2), 53-67. https://doi.org/10.17081/dege.9.2.2975

Valhondo, D. (2003). Gestión del conocimiento del mito a la realidad. Madrid: Díaz de Santos. 